\title{
Secretin-like Bioactivity in the Duodenal Mucosa in Patients with Peptic Ulcer and Ghronic Pancreatitis
}

\author{
Takaaki Takebe, Sozo Ishizuki, Toshitaka Kagaya, Masaru \\ Koizumi, Shigeki Kataoka, Tsutomu Kamei, Makoto Hanawa, \\ Susumu Takahashi, Kozo Ohyama, Katsuhiro Endo, Masanori \\ Mita and Akira Ishimori
}

The Third Department of Internal Medicine, Tohoku University School of Medicine, Sendai 980

Takebe, T., Ishrzuki, S., KagaYa, T., Kolzomi, M., Kataoka, S., Kamei, T., Hanawa, M., Takahashi, S., Ohyama, K., Endo, K., Mrta, M. and Ishimori, A. Secretin-like Bioactivity in the Duodenal Mucosa in Patients with Peptic Ulcer and Chronic Pancreatitis. Tohoku J. exp. Med., 1978, 126 (1), 13-26- Biopsy specimens of the duodenal mucosa were assayed to determine their secretin-like activity in 9 controls, 9 patients with gastric ulcer, 19 patients with duodenal ulcer, 4 patients with gastric and duodenal ulcer, and 13 patients with chronic pancreatitis. The bioassay of secretin was done on the pancreatic secretion in anesthetized rats. The sensitivity was in the order of $0.0625 \mathrm{CHR}$ unit/rat (4 $\mathrm{ng} / \mathrm{rat}$ ). In the range between 0.0625 and $0.5 \mathrm{CHR}$ units a satisfactory dose dependency was recognized. The following results were obtained. 1) The level of duodenal mucosal secretin-like activity in patients with gastric ulcer was the same as that in the controls, but was elevated in $32 \%$ of the patients with duodenal ulcer, $50 \%$ of those with gastric and duodenal ulcer, and $8 \%$ of those with chronic pancreatitis. 2) The high level of secretin-like activity noted in patients with duodenal ulcer was suspected to be related to the hypersecretion of gastric acid which is characteristic of this disease, but there was no correlation between gastric acid secretion and secretin-like activity in the duodenal mucosa. secretin; duodenal mucosa; peptic ulcer; chronic pancreatitis; bioassay

The upper part of the duodenum is a site of acid-base reaction, and the $\mathrm{pH}$ value of the site chiefly depends on the balance between $\mathrm{H}^{+}$cations of gastric juice and $\mathrm{HCO}_{3}^{-}$anions of pancreatic juice. Secretin produced by the mucous membrane of the upper small intestine indirectly participates in this neutralization reaction of the two ions. That is to say, secretin is liberated into the blood stream, as the intraduodenal $\mathrm{pH}$ value declines by the inflow of gastric acid into the duodenum. As a result, bicarbonate secreted by the pancreas neutralizes gastric acid, and inhibits the liberation of secretin with increasing intraduodenal $\mathrm{pH}$ value. Typical pathological conditions by which this acid neutralizing mechanism in the duodenum is disturbed are duodenal ulcer and chronic pancreatitis. The intraduodenal $\mathrm{pH}$ value is presumed to be lower than normal in both pathologies. The probable cause of

Received for publication, December $23,1977$. 
a low intraduodenal $\mathrm{pH}$ value is a relative excess of gastric $\mathrm{H}^{+}$cations in the former, while a relative shortage of pancreatic $\mathrm{HCO}_{3}{ }^{-}$anions in the latter. In recent years reports have been published that the development of duodenal ulcer is related to the disturbances of the acid neutralizing mechanism of the duodenum (Wormsley 1969) or disturbances of the mechanism for liberation of secretin into the blood stream (Bloom and Ward 1975), but as far as we know, no study has been done on the secretin content of duodenal mucous membrane. In this study, first, the bioassay system for the measurement of secretin activity was established, and then the secretin-like activity of duodenal mucosa obtained by biopsy from controls and patients with peptic ulcer or chronic pancreatitis was determined.

\section{Materials and Methods}

Subjects

The subjects of this study consisted of 9 controls, 9 patients with gastric ulcer, 19 patients with duodenal ulcer, 4 patients with gastric and duodenal ulcer, and 13 patients with chronic pancreatitis. By sex, the males outnumbered the females 41 to 13 , and their age ranged from 14 to 72 years. Of the controls 6 subjects had no abnormalities in the digestive system, while the remaining 3 had gastric diverticulum, duodenal polyp, and malabsorption syndrome (but pancreatic exocrine function was normal), respectively. The diagnosis of peptic ulcer was established by $\mathrm{x}$-ray examination and endoscopic examination of the upper gastrointestinal tract. When the duodenal mucous membrane was biopsied, ulcer was found in 13 subjects, ulcerative cicatrization in 17 subjects, and both in 2 subjects. Of the patients with chronic pancreatitis, 10 were diagnosed on the ground of pancreatic calcification and disorders of pancreatic exocrine function, and chronic pancreatitis was suggested by remarkable disturbance in the pancreatic function in the other 3 subjects. It was noted that 5 patients with chronic pancreatitis were complicated by moderate to severe diabetes mellitus.

\section{Methods}

Biopsy of the duodenal mucous membrane and isolation of secretin from biopsy materials

A duodenofiberscope of FDS (Machida) or JFB (Olympus) type was inserted into the second part of the duodenum of the subjects after fasting early in the morning, and 7 to 8 pieces of duodenal mucosa were obtained by biopsy forceps from the anterior and posterior walls at the same height as Vater's papillae.

The tissue specimens were placed into small lidded test tubes of polyethylene containing $450 \mu \mathrm{l}$ of $0.01 \mathrm{~N} \mathrm{HCl}$, and were immediately heated in a boiling water bath for $3 \mathrm{~min}$ in order to inactivate the proteolytic enzymes. After heating, $50 \mu \mathrm{l}$ of $1.0 \mathrm{~N} \mathrm{HCl}$ was added to the tissue specimens, and they were preserved at $-20^{\circ} \mathrm{C}$ until secretin was isolated. In isolating secretin, the frozen tissue specimens were thawed, and well homogenized in the polyethylene test tubes. The tissue specimens were then centrifuged at $10,000 \mathrm{rpm}$ at $4^{\circ} \mathrm{C}$, and the supernatant obtained was used in the bioassay. On the other hand, the sediment was well dried in a desiccator, and was weighed at the order of $0.01 \mathrm{mg}$.

\section{Bioassay of secretin}

Male rats of the Wistar strain weighing 260 to $580 \mathrm{~g}$ (average, $389 \mathrm{~g}$ ) were used in the bioassay of secretin. The animals were fasted for $24 \mathrm{hr}$ before the assay, only water was given freely. $47 \% \mathrm{w} / \mathrm{v}$ urethane was given intraperitoneally in a dose of $0.3 \mathrm{ml} / 100 \mathrm{~g}$ of body weight. In order to remove the tracheal contents, tracheotomy was performed. The femoral vein was exposed, and a PE 20 type polyethylene tube (I.D. 0.015 inch, Clay 
Adams, USA) was insented into the femoral vein for intravenous administration of standard secretin or test specimens. A small syringe filled with physiological saline was connected to the other end except during injection of secretin or specimens. The abdomen was opened through a midline incision, and the pyloric sphincter was ligated in order to avoid the liberation of endogenous secretin due to inflow of gastric acid into the duodenum. Then the common bile duct was ligated right above the duodenal opening, and the hepatic duct was exposed right under the hepatic porta, and a small incision was made into it. One PE 50 type polyethylene tube (I.D. 0.023 inch) was inserted into the hepatic duct in the direction of the hepatic porta, and another, in the direction of the duodenal opening. Both were fixed in position by ligation. The former was used to drain the bile, and the free end of the tube was left in the upper portion of the jejunum, while the latter was placed into the hepatic duct so as to draw pancreatic juice, and its other end was connected to a $1.0 \mathrm{ml}$ glass tube with graduations in $\mu 1$ units (Natsume Seisakusho Co., Japan). The glass tube was fixed $5 \mathrm{~cm}$ inferiorly from the back of the rat. Throughout the operation and bioassay procedure the animals were kept in warm with a 60-watt lamp to a rectal temperature of 34 to $36^{\circ} \mathrm{C}$.

Following the operation $100 \mathrm{CHR}$ units of CCK-PZ (Boots) and secretin (Boots) were dissolved in $4.0 \mathrm{ml}$ of physiological saline, and $200 \mu \mathrm{l}$ of the solution (5 CHR units of both hormones) was administered intravenously to the rats. The pancreatic duct was washed out with pancreatic juice drawn. The pancreatic juice with high content in protein that flowed out first was discarded, and the subsequent pancreatic secretion was introduced into the graduated glass tube. The output of pancreatic juice could be read down to $1 / 4 \mu l$, and recorded at 5 -min intervals. When 3 or more 5 -min ouputs were stabilized, it was taken as the basal pancreatic secretion.

Then, 50 CHR units of standard secretin (Eisai Co., Japan) was dissolved in $2.0 \mathrm{ml}$ of physiological saline, and $0.125,0.25$ and $0.5 \mathrm{CHR}$ units (and $0.03125,0.0625$ or $1.0 \mathrm{CHR}$ units as well where necessary) were administered intravenously. The output of pancreatic juice was recorded for $15 \mathrm{~min}$ immediately after administration and thereafter at 5-min intervals. When the 5 -min output was stabilized. it was recorded as the basal secretion. The pancreatic secreiton after administration of standard secretin was determined by subtracting the sixfold value of the average 5 -min basal secretion ( 30 -min basal secretion) from the total secretion for $30 \mathrm{~min}$ after intravenous administration of secretin, as shown in Table 1. Namely, the excess volume $(\mu \mathrm{l})$ was reported as the pancreatic secretion after administration of standard secretin.

Following the intravenous administration of at least three doses of standard secretin the logarithmic doses of secretin were plotted against the pancreatic secretion. When there was a linear relationship between the two variables, the test specimen was administered intravenously.

The doses of the specimen were so adjusted that the pancreatic secretory response to the specimen would fall within the range of its response to standard secretin. For the aforementioned number of biopsied materials the suitable dose for a single intravenous administration seemed to be in the order of 100 to $200 \mu l$. The pancreatic secretion after intravenous administration of the specimen was determined in the same way as after standard secretin administration (Table 1), and the secretin activities were determined from the dose response curve made by the standard secretin administration. The value thus obtained was divided by the dry weight of sediment obtained after isolation of secretin and expressed in CHR units per mg.

\section{Study of gastric acid secretion}

The basal gastric acid secretion for $60 \mathrm{~min}$ and the maximum gastric acid secretion for $60 \mathrm{~min}$ after stimulation with 3 - $\beta$-aminoethylpyrazole dihydrochloride in a dose of $1 \mathrm{mg} / \mathrm{kg}$ or t-amyloxycarbonyl tetrapeptide in a dose of $4 \mu \mathrm{g} / \mathrm{kg}$ were determined in 2 controls, 4 patients with gastric ulcer, 12 patients with doudenal ulcer, 3 patients with gastric and duodenal ulcer, and 7 patients with chronic pancreatitis, 28 subjects in total. 
TABLE 1. Pancreatic secretory response to standard secretin and extract of biopsied specimens

\begin{tabular}{cccc}
\hline Time (min) & $\begin{array}{c}0.5 \text { CHR unit } \\
\text { of secretin }\end{array}$ & \multicolumn{2}{c}{$\begin{array}{c}\text { Extract of biopsied specimens }(100 \mu \mathrm{l}) \\
\text { 1st injection }\end{array}$} \\
\hline-20 & 260.5 & & 2nd injection \\
-15 & $270.5 / 3.0^{*}$ & & \\
-10 & $273.5 / 3.0$ & & 383.5 \\
-5 & $276.5 / 3.0$ & 337.5 & $405.5 / 22.0$ \\
0 & 280.5 & $360.5 / 23.0$ & $413.0 / 7.5$ \\
15 & $303.0 / 22.5$ & $367.5 / 7.0$ & $418.0 / 5.0$ \\
20 & $311.0 / 8.0$ & $372.0 / 4.5$ & $421.5 / 3.5$ \\
25 & $316.0 / 5.0$ & $375.5 / 3.5$ & $425.5 / 4.0$ \\
30 & $320.0 / 4.0$ & $379.0 / 3.5$ & $429.5 / 4.0$ \\
35 & $323.5 / 3.5$ & & $433.0 / 3.5$ \\
40 & $327.0 / 3.5$ & & $436.0 / 3.0$ \\
45 & $329.0 / 2.0$ & & \\
50 & $332.0 / 3.0$ & & \\
\hline
\end{tabular}

*A/B A, read of scale on collecting tube $(\mu \mathrm{l}) ; \mathrm{B}$, volume of pancreatic juice $(\mu \mathrm{l})$. Calculation $=$ total volume of pancreatic juice for $30 \mathrm{~min}$ after injection $-6 \times$ mean of basal secretion for $5 \mathrm{~min}$ before and after injection.

Response to $0.5 \mathrm{CHR}$ unit of secretin $=(22.5+8.0+5.0+4.0)-6 \times 3.0=21.6 \mu \mathrm{l}$.

Response to extract of biopsied specimens (1st injection $)=(23.0+7.0+4.5+3.5)-6$ $\times 3.0=20.0 \mu l$.

Response to extract of biopsied specimens (2nd injection $)=(22.0+7.5+5.0+3.5)-6$ $\times 3.0=20.0 \mu 1$.

\section{Study of pancreatic bicarbonate secretion}

Pancreatic exocrine function was investigated by the duodenal drainage method in 2 controls, 1 patient with gastric ulcer, and 13 patients with chronic pancreatitis. In this investigation secretin was administered in a dose of $1.2 \mathrm{CHR}$ units $/ \mathrm{kg} / \mathrm{hr}$ (submaximum dose) for $60 \mathrm{~min}$, and the maximum 60-min pancreatic secretion of bicarbonate was calculated on the basis of finding that the bicarbonate output increased $60 \%$ when the dose of $1.2 \mathrm{CHR}$ units $/ \mathrm{kg} / \mathrm{hr}$ was increased to $6.0 \mathrm{CHR}$ units $/ \mathrm{kg} / \mathrm{hr}$ (approximately maximum dose, unpublished).

\section{RESULTS}

Dose response of pancreatic secretion to standard secretin in rats

Standard secretin was administered intravenously in doses of $0.125 \mathrm{CHR}$ unit in 3 rats, 0.25 in 6 and 0.5 in 7 , and the pancreatic secretion was determined at 5 min intervals. The secretion reached a peak $10 \mathrm{~min}$ after the administration of standard secretin at all the dose levels and thereafter declined to the basal level in about $30 \mathrm{~min}$ (Fig. 1).

In order to determine the influence of rat body weight on the dose response of pancreatic exocrine function to secretin, another group of rats were divided into four subgroups according to the body weight, as shown in Table 2 , and their pancreatic secretion after administration of secretin in doses of $0.125,0.25$ and $0.5 \mathrm{CHR}$ units was determined. There proved no significant differences in dose response of the mean secretion among the four subgroups of rats. The coefficients of variation of 


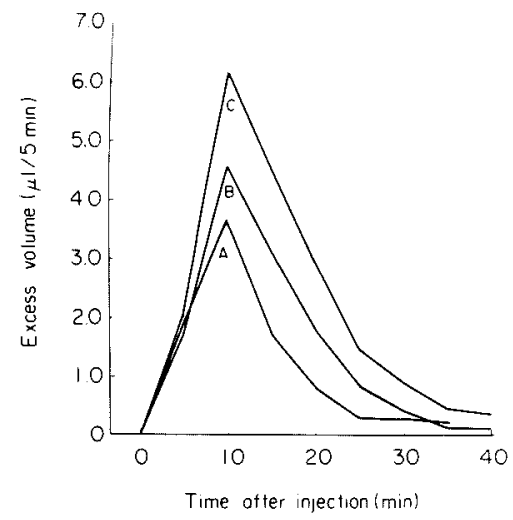

Fig. 1. The effect of secretin on the flow rate of pancreatic juice in rats.

A : 0.125 CHR unit $(n=3)$. B: 0.25 CHR unit $(n=6) . \quad$ C: 0.5 CHR unit $(n=7)$.

TABLE 2. The influence of body weight on the pancreatic secretory response in the rats

\begin{tabular}{|c|c|c|c|c|}
\hline $\begin{array}{l}\text { Body weight of } \\
\text { rats }(\mathrm{g})\end{array}$ & & $0.125 \mathrm{CHR}$ unit & 0.25 CHR unit & 0.5 CHR unit \\
\hline \multirow{3}{*}{$200-290$} & $\begin{array}{l}\text { Number } \\
\text { of rats }\end{array}$ & 4 & 4 & 4 \\
\hline & Mean & 10.84 & 15.19 & 19.59 \\
\hline & S.D. & 3. 184 & 3.295 & 2. 805 \\
\hline \multirow{3}{*}{$300-390$} & $\begin{array}{l}\text { Number } \\
\text { of rats }\end{array}$ & 16 & 18 & 18 \\
\hline & Mean & 10.73 & 15.22 & 21.18 \\
\hline & S.D. & 2.36 & 4. 183 & 5.359 \\
\hline \multirow{3}{*}{$400-490$} & $\begin{array}{l}\text { Number } \\
\text { of rats }\end{array}$ & 13 & 14 & 14 \\
\hline & Mean & 8.27 & 13. 23 & 19.28 \\
\hline & S.D. & 2.47 & 3. 712 & 5.679 \\
\hline \multirow{3}{*}{$500-590$} & $\begin{array}{l}\text { Number } \\
\text { of rats }\end{array}$ & 4 & 4 & 4 \\
\hline & Mean & 9.91 & 17.41 & 24.56 \\
\hline & s.D. & 2.88 & 4.092 & 3. 819 \\
\hline \multirow{4}{*}{ Total } & $\begin{array}{l}\text { Number } \\
\text { of rats }\end{array}$ & 37 & 40 & 40 \\
\hline & Mean & 9.79 & 14.74 & 20.69 \\
\hline & S.D. & 2.803 & 4. 135 & 5. 373 \\
\hline & Vc. $(\%)$ & 28.6 & 28.1 & 26.0 \\
\hline
\end{tabular}

s.D., standard deviation; Vc., coefficient of variation.

pancreatic secretion for doses of $0.125,0.25$ and 0.5 CHR units of secretin were 28.6 $\%, 28.1 \%$ and $26.0 \%$, respectively, in all the rats.

The dose response relationship between pancreatic secretion and secretin was studied in the same rats, repeating its administration once or more. The reproducibility of the dose response thus determined is presented in Table 3 . The coefficients of variation of pancreatic secretion after repeated administrations of 
secretin in doses of $0.125,0.25$ and $0.5 \mathrm{CHR}$ units were obtained in individual rats, and averaged. The mean coefficients of variation were $6.7 \%, 7.9 \%$ and $4.6 \%$, respectively.

TABLE 3. The reproducibility in the response of rat's pancreas to various doses of secretin

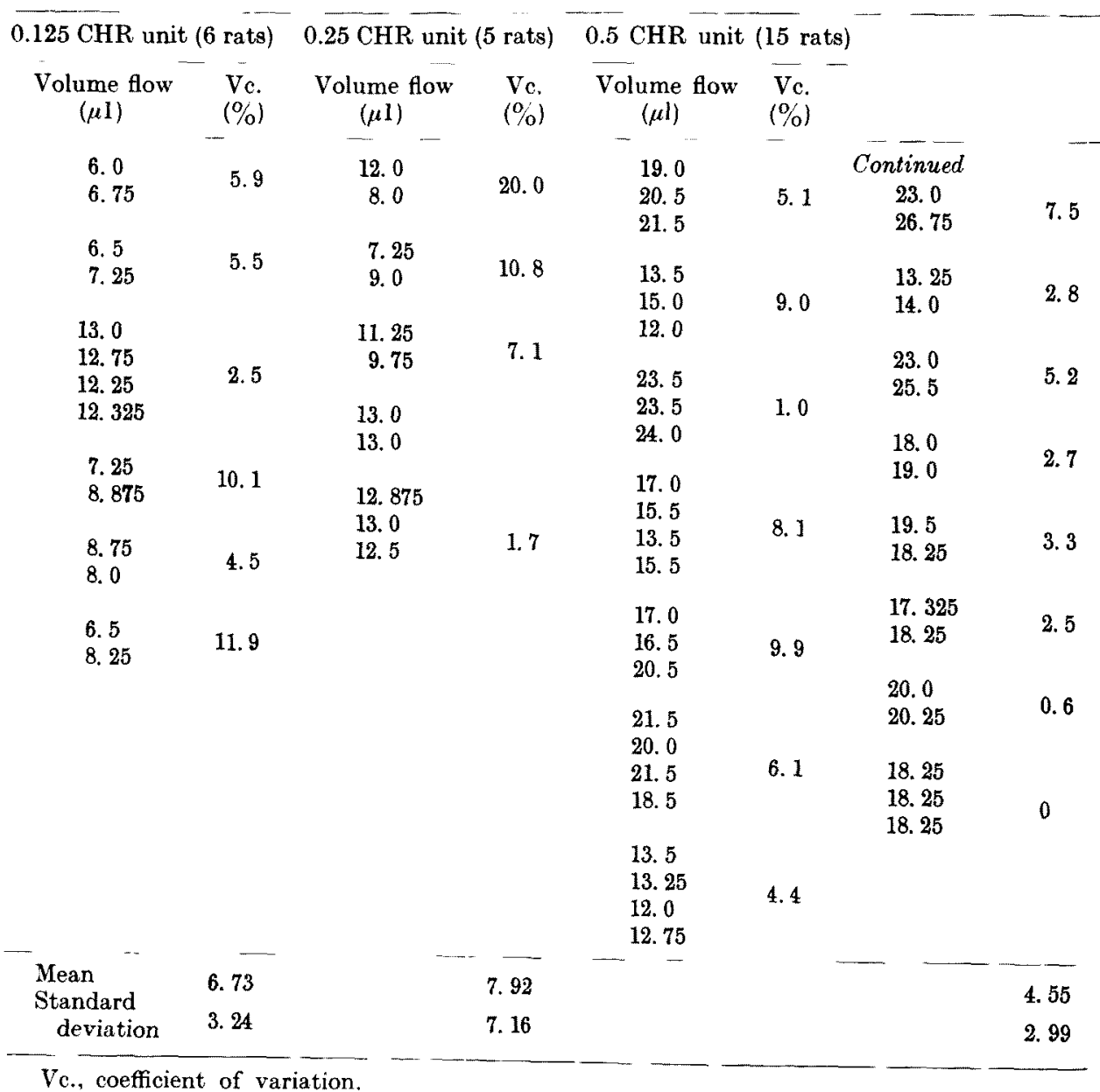

Standard secretin was administered intravenously to 40 rats in doses of 0.03125 $0.0625,0.125,0.25,0.5$ and $1.0 \mathrm{CHR}$ units 207 times in total, and the dose response curve was plotted, laying off the logarithmic dose along the horizontal axis and the excess volume along the vertical axis, as shown in Fig. 2. As is clear from this figure, there was a close correlation between logarithmic dose of secretin and pancreatic secretion $(r=0.8163)$, and its regression line was expressed by equation $Y=14.5 X+23.4$. Especially in the dose range of 0.0625 to $0.5 \mathrm{CHR}$ units there was a linear relationship between the two variables. 


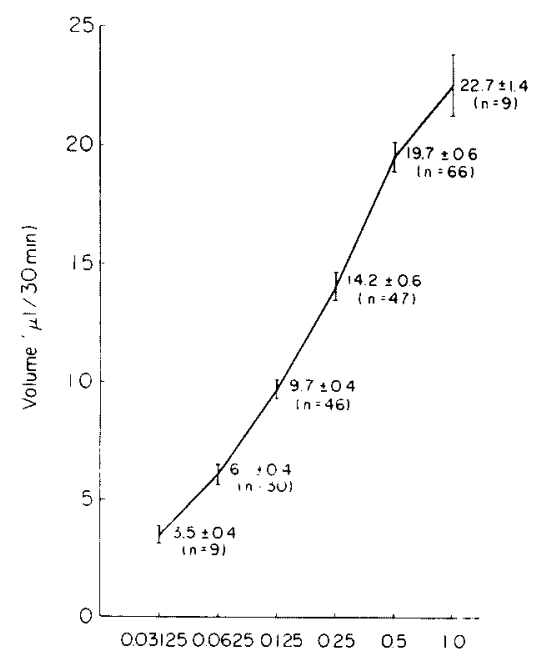

Fig. 2. The relation between logarithmic dose of secretin and pancreatic secretion. Each value is expressed as mean \pm S.D.

Duodenal mucosal secretin-like activity in controls and patients

The duodenal mucosal secretin-like activity in the controls ranged from 0.1496 to $0.4305 \mathrm{CHR}$ units/mg of dry weight, and its mean value and coefficient of variation were $0.2836 \mathrm{CHR}$ unit/mg and $36 \%$ (Fig. 3). When its normal range was defined by the mean $+2 \times$ standard deviation, it was between 0.0784 and 0.4888 CHR units/mg.

The duodenal mucosal secretin-like activity in the group of patients with gastric ulcer was in the range of 0.1433 to $0.4218 \mathrm{CHR}$ units $/ \mathrm{mg}$. No patient of this group was outside the above-mentioned normal range. The mean value of this group was $0.2508 \mathrm{CHR}$ unit/mg, nearly equal to that of the control group (Fig. 3).

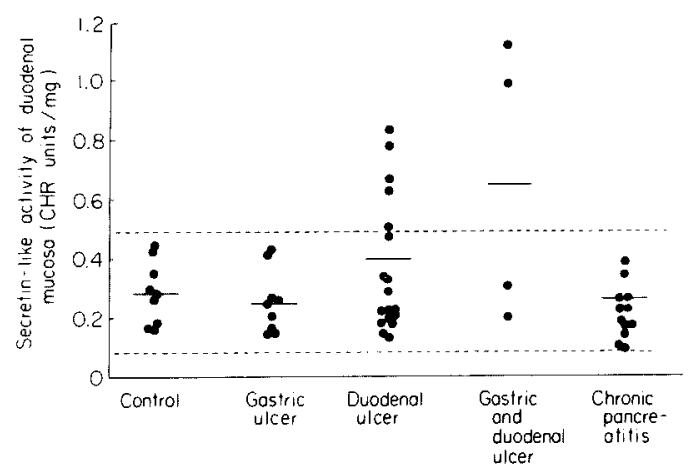

Fig. 3. The secretin-like activity of duodenal mucosa in control subjects and patients with various diseases. The bars give mean values. The dotted lines indicate mean \pm 2 s.D. 
The duodenal mucosal secretin-like activity in the group of patients with gastric and duodenal ulcer was in the range of 0.1333 to $1.059 \mathrm{CHR}$ units $/ \mathrm{mg}$. No patient of this group showed a value lower than the normal range, but $6(32 \%)$ of 19 patients showed values exceeding the normal range. The mean value of this group was 0.3994 CHR unit/mg, slightly higher than that of the control group, but there was no significant difference between the means of the two groups (Fig. 3).

The duodenal mucosal secretin-like activity in the group of patients with gastric and duodenal ulcer was in the range of 0.197 to $1.133 \mathrm{CHR}$ units $/ \mathrm{mg}$. Two of 4 patients showed values exceeding the normal range. The mean value of this group was $0.6553 \mathrm{CHR}$ unit/mg and was significantly higher than the corresponding value of the control group $(p<0.05)$ (Fig. 3 ).

The mean value of duodenal mucosal secretin-like activity of all the patients with peptic ulcer was $0.39 \mathrm{CHR}$ unit/mg, slightly higher than that of the control group, but the difference was not statistically significant. When the duodenal mucosal secretin-like activity was determined in 23 patients with duodenal ulcer regardless of complication by gastric ulcer, their mean value was $0.444 \mathrm{CHR}$ unit/mg, and did not significantly differ from the corresponding value of the control group. These patients were divided into a group with ulcer and a group with ulcerative cicatrization according to the findings obtained with the duodenofiberscope. The mean values of duodenal mucosal secretin-like activity of the two groups were 0.4154 and $0.459 \mathrm{CHR}$ units/mg, respectively.

The duodenal mucosal secretin-like activity in the group of patients with chronic pancreatitis was in the range of 0.0785 to $0.8836 \mathrm{CHR}$ units $/ \mathrm{mg}$. No patient of this group showed a value lower than the normal range, but 1 of 13 patients showed a value exceeding the normal range. The mean value of this group was $0.265 \mathrm{CHR}$ unit/mg, nearly equal to that of the control group (Fig. 3).

Gastric acid secretion and pancreatic bicarbonate secretion

Gastric acid secretion could be determined in 28 of the subjects of this study, and the basal gastric acid secretion, maximum gastric acid secretion, and duodenal mucosal secretin-like activity were also determined in the 28 subjects, as summarized in Table 4. When their maximum gastric acid secretion was specifically compared with the normal ranges $(9.2-17.8 \mathrm{mEq} / \mathrm{hr}$ for males and $3.5-14.9 \mathrm{mEq} / \mathrm{hr}$ for females) established at our laboratory, the following results were obtained. One subject of the control group showed a normal value, and one a lower value than normal; 3 subjects of the group with gastric ulcer gave a normal value, and 1 a higher value than normal; 2 of the group with duodenal ulcer, no matter whether complicated by gastric ulcer or not, gave a normal value, 12 a higher value than normal, and 1 a lower value than normal; and 1 of the group with chronic pancreatitis gave a normal value, 2 a higher value than normal, and 4 a lower value than normal. As a result, $80 \%$ of 15 patients with duodenal ulcer had an increase in maximum gastric acid secretion, whereas $57 \%$ of 7 patients with chronic pancreatitis had a decrease in maximum gastric acid secretion. 
TABLE 4. Gastric acid output and secretin-like activity of duodenal mucosa

\begin{tabular}{|c|c|c|c|c|c|c|}
\hline Cases & Sex & Age & Group & $\begin{array}{l}\text { Basal acid } \\
\text { output } \\
\text { (mEq/hr) }\end{array}$ & $\begin{array}{l}\text { Maximum } \\
\text { acid output } \\
\text { (mEq/hr) }\end{array}$ & $\begin{array}{l}\text { Secretin-like activity } \\
\text { of duodenal mucosa } \\
\text { (CHR units/mg) }\end{array}$ \\
\hline T.G. & $\mathrm{F}$ & 44 & Control & 0.48 & 6.26 & 0.4305 \\
\hline K. $\mathrm{O}$. & $\mathbf{M}$ & 63 & Control & 0.12 & 5.82 & 0.1629 \\
\hline M.K. & $\mathbf{F}$ & 31 & G.U. & 2. 52 & 13.34 & 0.2446 \\
\hline H.K. & $\mathbf{F}$ & 39 & G.U. & 1.12 & 7.7 & 0.1433 \\
\hline Y.Y. & $\mathbf{F}$ & 43 & G.U. & 9. 54 & 16.92 & 0.4126 \\
\hline T.O. & M & 71 & G.U. & 1.14 & 14.78 & 0.2631 \\
\hline S.H. & M & 14 & D.U. & 8.75 & 18.64 & 0.2215 \\
\hline K.S. & $\mathbf{M}$ & 20 & D.U. & 17.52 & 30.59 & 0.2185 \\
\hline Y.O. & $\mathbf{M}$ & 20 & D.U. & 12.7 & 31.22 & 0.3405 \\
\hline T.A. & $\mathbf{M}$ & 24 & D.U. & 30.28 & 50.1 & 0.5 \\
\hline M.I. & $\mathrm{F}$ & 25 & D.U. & 7.06 & 16.86 & 0.7784 \\
\hline K.N. & $\mathbf{F}$ & 25 & D.U. & 5. 59 & 11. 68 & 0.1993 \\
\hline T.H. & $\mathbf{M}$ & 26 & D.U. & 9.06 & 21.35 & 0.3234 \\
\hline T.S. & M & 29 & D.U. & 15.31 & 39.6 & 0.1459 \\
\hline A.F. & $\mathbf{M}$ & 41 & D.U. & 6.34 & 29.07 & 0.2295 \\
\hline K.Y. & M & 43 & D.U. & 4.56 & 15.14 & 0.286 \\
\hline M.S. & M & 62 & D.U. & 6.38 & 34.7 & 0.1962 \\
\hline S.S. & $\mathbf{M}$ & 63 & D.U. & 4.7 & 28.9 & 0.1333 \\
\hline H.K. & $\mathbf{M}$ & 38 & G.U. + D.U. & 4. 44 & 31.2 & 0.9893 \\
\hline T.I. & M & 40 & G.U.+D.U. & 2.51 & 18.66 & 0.302 \\
\hline T.I. & $\mathbf{M}$ & 65 & G.U.+D.U. & 0 & 0 & 0.197 \\
\hline S.S. & M & 38 & C.P. & 3.59 & 32.4 & 0.187 \\
\hline A.Y. & M & 42 & C.P. & 1.72 & 4. 75 & 0.1614 \\
\hline H.M. & M & 48 & C.P. & 0 & 1. 54 & 0.2529 \\
\hline T.F. & M & 54 & C.P. & 0.05 & 3.21 & 0.261 \\
\hline G.K. & M & 55 & C.P. & 1.6 & 21.06 & 0.2222 \\
\hline K.T. & $\mathbf{M}$ & 56 & C.P. & 0.38 & 17.39 & 0.8836 \\
\hline A.Y. & M & 64 & C.P. & 0 & 0 & 0.3467 \\
\hline
\end{tabular}

G.U., gastric ulcer; D.U., duodenal ulcer; C.P., chronic pancreatitis.

TABLE 5. Gastric acid output, pancreatic bicarbonate output and secretin-like activity of duodenal mucosa

\begin{tabular}{llclccc} 
Cases & Sex & Age & Group & $\begin{array}{c}\text { Maximum } \\
\text { acid output } \\
\text { (mEq/hr) }\end{array}$ & $\begin{array}{c}\text { Calculated maximum } \\
\text { pancreatic bicarbonate } \\
\text { output (mEq/hr) }\end{array}$ & $\begin{array}{c}\text { Secretin-like activity } \\
\text { of duodenal mucosa } \\
\text { (CHR units/mg) }\end{array}$ \\
\hline T.G. & F & 44 & Control & 6.26 & 16.84 & 0.4305 \\
M.K. & F & 31 & G.U. & 13.34 & 39.78 & 0.2446 \\
S.S. & M & 38 & C.P. & 32.4 & 19.55 & 0.187 \\
A.Y.* & M & 42 & C.P. & 4.75 & 3.9 & 0.1614 \\
H.M.* & M & 48 & C.P. & 1.54 & 0.24 & 0.2529 \\
T.F. & M & 54 & C.P. & 3.21 & 2.22 & 0.261 \\
G.K.* & M & 55 & C.P. & 21.06 & 9.52 & 0.2222 \\
K.T. & M & 56 & C.P. & 17.39 & 2.64 & 0.8836 \\
A.Y.* & M & 64 & C.P. & 0 & 6.51 & 0.3467 \\
\hline
\end{tabular}

G.U., Gastric ulcer; C.P., Chronic pancreatitis. *Alcoholics.

Pancreatic exocrine function could be determined in only a small number of subjects, the maximum pancreatic bicarbonate secretion, maximum gastric acid secretion, and duodenal mucosal secretin-like activity were determined in these 
subjects, as shown in Table 5. Two subjects of the control group and 1 of the group with gastric ulcer showed a normal value of maximum pancreatic bicarbonate secretion, while all patients with chronic pancreatitis showed values pointing to pancreatic impairement.

\section{Discussion}

Various species of animals are used in the bioassay of secretin. The procedure using anesthetized rats for this purpose was developed by Love (1957), and its usefulness was recognized by Heatley (1968a) and Tachibana (1971) in later years. As there is a report that the response of pancreatic exocrine function in anesthetized rats to secretin is more marked in males than in females (Heatley 1968a), male rats of the Wistar strain were chosen for this study. As the cannulation of the hepatic duct was difficult in rats weighing less than $300 \mathrm{~g}$, ones of mainly 300 to $400 \mathrm{~g}$ were used in this study. According to Tachibana (1971), pancreatic secretion per rat stimulated with secretin was influenced by the body weight of the rat used, whereas the results obtained in this study indicated that the influence of the rat body weight was negligible, so long as the dose of secretin was within the limits of 0.125 to 0.5 CHR units/rat (Table 2). However, the inter-rat variations were $26 \%$ to $29 \%$. The rather high variability of measurement may be ascribed to several factors, such as varying level of anesthesia and technique of operation.

In bioassay, generally speaking, it is important to achieve a high reproducibility of the result obtained at the same dose in the same subjects and a high dose dependency of the result. In the assay system described in this paper the reproducibility of the dose response of pancreatic secretion to secretin in the dose range of 0.125 to $0.5 \mathrm{CHR}$ unit was rather high, as shown in Table 3. Pancreatic secretion may fluctuate during assay for various causes, such as an increase in the viscosity of pancreatic juice, obstruction of the flow of pancreatic juice due to collective tube twisting, changes in body temperature, and varying level of anesthesia. To eliminate such undesired factors care should be used to wash out highly viscous pancreatic juice in the pancreatic duct with the juice secreted right after administration of secretin in a relatively high dose following the operational procedure, to collect all pancreatic juice, to maintain the related organs at a constant temperature throughout the assay, and to complete the assay within 10 hr of anesthetization.

There are reports that pancreatic secretion in anesthetized rats is dependent on the actual dose of secretin (Love 1957) or the logarithmic dose (Heatley 1968a; Tachibana 1971). The results obtained in this study coincide with the latter of the two different reports (Fig. 2). The measurement limit in this assay system is approximately $4 \mathrm{ng} / \mathrm{rat}$, as $16,000 \mathrm{CHR}$ units of the preparation supplied as standard secretin is equivalent to $1 \mathrm{mg}$.

Tachibana (1971) recommends a twin crossover design as a technique for determination of the secretin bioactivity in unknown specimens. However, when no 
more than 7 to 8 pieces of the tissue of the duodenal mucosa are used as in this study, the twin crossover design is hard to employ for want of specimens. To compensate this study for specimen insufficiency, at least 3 doses of standard secretin were chosen, and the doses of specimen were so settled under the condition favoring the dose dependency of pancreatic secretion to the 3 doses of secretin that the reactions of the pancreatic output to the specimen would fall within the range of its response to standard secretin.

The $\mathrm{HCl}$ extract of the duodenal mucous membrane is presumed to contain not only secretin but various other substances. When the isolated guinea pig ileum and rat cardiac orifice were used for bioassay of histamine and serotonin, respectively, extract of 7 to 8 pieces of biopsy materials obtained from the duodenal mucosa contained about $4 \mu \mathrm{g}$ of histamine and $0.28 \mu \mathrm{g}$ of serotonin. At these levels of histamine and serotonin, however, they have little chance of stimulating pancreatic secretion. On the other hand, acetylcholine was not detected in these tissue specimens. However, as the extract of the tissue specimens contained a substance that caused the guinea pig gallbladder to contract, it was beyond doubt that they contained CCK-PZ.

CCK-PZ is known to stimulate pancreatic secretion in the anesthetized rat alone and to produce a synergistic effect on the secretin activity (Heatley 1968a, b; Tachibana 1971). As the stimulation of pancreatic secretion by crude secretin extract diminishes after repeated administration, CCK-PZ produces a synergistic effect with secretin but not without tachyphylaxis. In this study, therefore, the pancreatic duct was washed out with administration of crude secretin extract (supplied by the laboratories of Eisai Co.) or a mixture of secretin (Boots) and CCKPZ (Boots) in a relatively large dose after operation. As a result, the decline in pancreatic secretion after repeated administration of the specimen could be prevented (Table 1). In the bioassay, however, where pancreatic secretion in the rats was studied, the stimulant effects of both secretin and CCK-PZ (probably the additive effects of both hormones) were reflected at the same time. Therefore, the pancreatic stimulant effect of the bioassay specimen can reasonably be described as secretin-like activity. As water contained in or adhering to the biopsy specimen was thought to account for a significant part of its total weight, its dry weight was used. It should also be noted that $0.1 \mathrm{~N} \mathrm{HCl}$ used in the extraction of secretin from the biopsy specimen had no stimulant effect on pancreatic secretion.

When the duodenal mucosal secretin-like activity was determined in the control group and groups of patients with various diseases of the digestive system, there were little differences among the control group, group with gastric ulcer, and the group with chronic pancreatitis. On the other hand, the duodenal mucosal secretin-like activity was elevated in $8(35 \%)$ of 23 patients with duodenal ulcer or duodenal ulcerative cicatrization, no matter whether or not it was complicated by gastric ulcer as shown in Fig. 3. The duodenal mucosal secretin-like activity was checked in each patient for relations with non-specific factors, such as sex, age, dry weight of biopsy specimen, and period between biopsy and assay, but 
no relations were revealed between them. Accordingly, the tendency toward elevated duodenal mucosal secretin-like activity in patients with duodenal ulcer was presumed to be specific to this disease. Furthermore, this tendency was present in the patients regardless of the character of duodenal ulcer and the duration of the disease.

The mechanism for development of duodenal ulcer is not clear enough, but the action of excess gastric acid on the duodenal mucous membrane and disorders of the mucous membrane defense or restoring mechanism are thought to have something to do with the development of duodenal ulcer. Wormsley (1969) examined the neutralization of hydrochloric acid after its injection into the duodenum and found that less $\mathrm{HCl}$ was neutralized by pancreatic bicarbonate in patients with duodenal ulcer than in normal persons and patients with gastric ulcer. In concluding his paper Wormsley (1969) inferred that this phenomenon was related to the development of ulcer. According to Demling (1969) who also had recourse to duodenal acidification in an attempt to clarify the mechanism for development of duodenal ulcer, the neutralization of acid in the duodenum and the response of pancreatic secretion to exogenous secretin did not differ from normal persons to patients with duodenal ulcer. Bloom and Ward (1975) determined the blood levels of immunoreactive secretin in normal persons and patients with duodenal ulcer before and after injection of $\mathrm{HCl}$ into the duodenum, and made the inference from the results of their study that the liberation of endogenous secretin into the blood stream was disturbed after injection of $\mathrm{HCl}$ in patients with duodenal ulcer. On the other hand, Isenberg et al. (1977) reported that the liberation of endogenous secretin into the blood stream did not differ from normal persons in patients with duodenal ulcer. Be that as it may, this hormone contributes a great deal to the neutralization of acid in the duodenum by promoting the pancreatic and hepatic secretions of bicarbonate and by inhibiting the stimulation of gastric acid secretion by gastrin. The studies on the production of secretin in the mucous membrane of the upper small intestine, its liberation into the blood stream, and the sensitivity of the target cell to this hormone in patients with duodenal ulcer are very important to the clarification of the mechanism for development of this disease and its treatment.

Two possible causes for an increase in duodenal mucosal secretin-like activity noted in several patients with duodenal ulcer were inferred from the results of consideration of the literature as follows: One possibility is that the hormone stored in the mucous membrane increased as a result of disturbance of the liberation of secretin into the blood stream as in one disposition of patients with duodenal ulcer described by Bloom and Ward (1975), the other possibility is that the liberation was stimulated by excess $\mathrm{H}^{+}$cations in the duodenum so that the production of the hormone in the mucous membrane was promoted. With especially the latter possibility in mind, the relationship between maximum gastric acid secretion and duodenal mucosal secretin-like activity was studied. As a result, the maximum gastric acid secretion was elevated in 3 of 4 patients with 
elevated secretin-like activity (Table 5). As shown in Fig. 4, however, there was no correlation between the two parameters when logarithmic maximum gastric acid secretion was plotted against duodenal mucosal secretin-like activity.

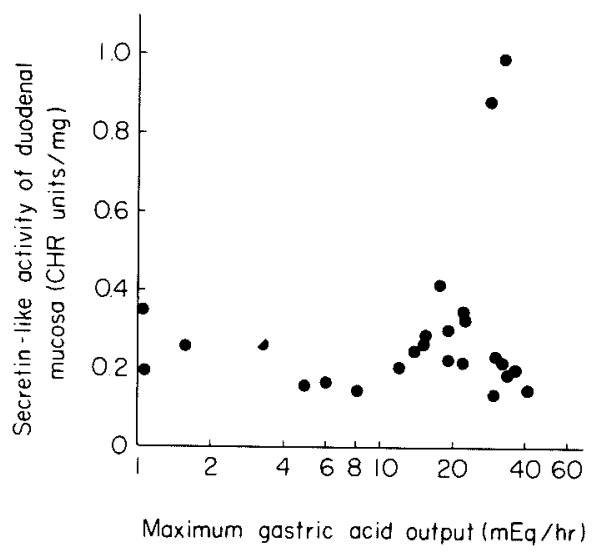

Fig. 4. Maximum gastric acid output and secretin-like activity of duodenal mucosa.

The activity of the digestive hormone acting on the damaged target cell is of interest. From this viewpoint, chronic pancreatitis was included in this study. It is presumed that the duodenal acid-base balance is inclined toward acidity in chronic pancreatitis due to a remarkable decline in pancreatic secretion of bicarbonate. For this reason the duodenal mucosal secretin-like activity in chronic pancreatitis was expected to show the same tendency as in duodenal ulcer, but the activity was elevated in only one of 13 patients with chronic pancreatitis. When the gastric acid-bicarbonate balance was compared with the duodenal mucosal secretin-like activity, bicarbonate secretion was remarkably decreased in one patient with non-alcoholic chronic pancreatitis (case K.T. in Table 5), but in this Case the gastric acid secretion was normal and duodenal mucosal secretin-like activity was elevated. In 3 patients with alcoholic chronic pancreatitis, however, both bicarbonate secretion and gastric acid secretion were decreased, and presumably for this reason the duodenal mucosal secretin-like activity was within the normal limits.

At the present moment it is difficult to conclude whether the increase in duodenal mucosal secretin-like activity noted mainly in patients with duodenal ulcer was a primary or secondary manifestation of this disease. To clarify this phenomenon further studies must be conducted in the same subjects concerning the contents of hormones in the duodenal mucous membrane, blood levels of hormones, and neutralization after duodenal acidification.

\section{Acknowledgment}

This study was supported in part by the Chronic Pancreatitis Research Grant of the Ministry of Health and Welfare of Japan. 


\section{References}

1) Bloom, S.R. \& Ward, A.S. (1975) Failure of secretin release in patients with duodenal ulcer. Brit. med. J., 18, 126-127.

2) Demling, L. (1969) Neutralizing capacity of the pancreas. In: Non-Insulin-Producing Tumors of the Pancreas, edited by L. Demling \& R. Ottenjann, Georg Thieme Verlag, Stuttgart, pp. 200-213.

3) Heatley, N.G. (1968a) The assay of secretin in the rat. J. Endocr., 42, 535-547.

4) Heatley, N.G. (1968b) The assay of pancreozymin, and of secretin and pancreozymin simultaneously, in the rat. $J$. Endocr., 42, 549-557.

5) Isenberg, J.I., Cano, G. \& Bloom, S.R. (1977) Effect of graded amounts of acid instilled into the duodenum on pancreatic bicarbonate secretion and plasma secretin in duodenal ulcer patients and normal subjects. Gastroenterology, 72, 6-8.

6) Love, J.W. (1957) A method for the assay of secretin, using rats. Quart. J. exp. Physiol., 42, 279-284.

7) Tachibana, S. (1971) The bioassay of secretin in the rat. Jap. J. Pharmacol., 21, 325-336.

8) Wormsley, K.G. (1969) Response to duodenal acidification in man - 1. Electrolyte changes in the duodenal aspirate. Scand. J. Gastroent., 4, 717-726. 